\title{
A Coordinated Tiling and Batching Framework for Efficient GEMM on GPUs
}

\author{
Xiuhong $\mathrm{Li}^{1}$, Yun Liang ${ }^{1, *}$, Shengen $\mathrm{Yan}^{2}$, Liancheng Jia ${ }^{1}$, Yinghan $\mathrm{Li}^{2}$ \\ ${ }^{1}$ Center for Energy-efficient Computing and Applications, School of EECS, Peking University \\ ${ }^{2}$ SenseTime Incorporation \\ \{lixiuhong,ericlyun,jlc\}@pku.edu.cn,\{yanshengen,liyinghan\}@sensetime.com
}

ACM Reference format:

Xiuhong $\mathrm{Li}^{1}$, Yun Liang ${ }^{1}$, , Shengen $\mathrm{Yan}^{2}$, Liancheng Jia ${ }^{1}$, Yinghan $\mathrm{Li}^{2}$. 2019. A Coordinated Tiling and Batching Framework for Efficient GEMM on GPUs. In Proceedings of 24th ACM SIGPLAN Symposium on Principles and Practice of Parallel Programming, Washington, DC, USA, February 16-20, 2019 (PPoPP '19), 2 pages.

https://doi.org/10.1145/3293883.3295734

\section{A Artifact Appendix}

\section{A.1 Abstract}

This section is mainly the guideline to perform artifact evaluation for this paper. We first describe the directory tree of our code, which contains the code of both related work and our work. Then, we present the check-list for the evaluation. Finally, experiment workflow shows how to access the source code and how to use the scripts to perform detailed validation.

\section{A.2 Description}

The following is the directory tree of the code, which contains eight sub-directories as follows:

- data. In this sub-directory, we provide a gen_data binary to generate the data-set used in the following evaluation.

- include. This sub-directory contains a header file for CUDA run-time, cuBLAS and cuDNN error check.

- default. This sub-directory contains the source code for default execution.

- cke. This sub-directory contains the source code for concurrent kernel execution using stream interface.

- magma. This sub-directory contains the source code of the batched GEMM implementation proposed in MAGMA paper.

${ }^{*}$ Corresponding author

Permission to make digital or hard copies of all or part of this work for personal or classroom use is granted without fee provided that copies are not made or distributed for profit or commercial advantage and that copies bear this notice and the full citation on the first page. Copyrights for components of this work owned by others than ACM must be honored. Abstracting with credit is permitted. To copy otherwise, or republish, to post on servers or to redistribute to lists, requires prior specific permission and/or a fee. Request permissions from permissions@acm.org.

PPoPP '19, February 16-20, 2019, Washington, DC, USA

(c) 2019 Association for Computing Machinery.

ACM ISBN 978-1-4503-6225-2/19/02 ..\$15.00

https://doi.org/10.1145/3293883.3295734
- tiling. This sub-directory contains the source code of the tiling engine proposed in our paper.

- batching. This sub-directory contains the source code of the batched GEMM implementation incorporating both of the tiling engine and batching engine in our paper.

- google-net_cudnn. This sub-directory contains the source code of a Google-Net inference framework built by cuDNN APIs and our batched GEMM techniques.

\section{A.3 Artifact check-list}

- Algorithm: Multiple variations of batched-GEMM on GPUs.

- Program: CUDA and $\mathrm{C} / \mathrm{C}++$ code.

- Compilation: nvec 9.0 with -O3 flag.

- Binary: CUDA executable.

- Data set: random matrix size and batch size.

- Run-time environment: Ubuntu 16.04 with CUDA SDK 9.0 installed.

- Hardware: Any GPUs with compute capability $>=5.0$ (Recommended GPU: NVIDIA V100 GPU.)

- How much disk space required (approximately)?: $<=10 \mathrm{Mb}$

- How much time is needed to prepare workflow (approximately)?: $<=30$ minutes

- How much time is needed to complete experiments (approximately)?: <=30 minutes

- Publicly available?: Yes.

\section{A.4 Experiment workflow}

For the convenience of the artifact evaluation, we only provide a few simple scripts in each sub-directory. Below are the steps to download our code, run the experiments, and observe the results.

\section{A.4.1 Download the code.}

We provide two methods to obtain the code.

\$git clone ppopp_ar@scc.eescer.com:/pub/tiling_batching_gemm.git

The password is 123456 . Note that there is no dot in the password.

\section{A.4.2 Build and Run the experiments.}

Comparison. There are five variants: default, cke, and magma in related works, as well as tiling and batching in our paper. In these five sub-directories, they all contain a run.sh script and you can run it to obtain the results.

Google-net Real-world case study. We provide two different baseline implementations. We can compare the baseline version with 
convolution based on batched-GEMM proposed in this paper. They can be switched by modifying the variable USE_MULTI_STREAM in Makefile. 